\title{
RCT on molar replacement with wide diameter implants: to place the implant immediately or to wait 4 months after socket preservation?
}

\author{
Erta Xhanari2, Marco Tallarico ${ }^{1}$, Silvio Mario Meloni ${ }^{3}$
}

${ }^{1}$ Private practice, Rome, Italy; ${ }^{2}$ Private practice, Tirana, Albania; ${ }^{3}$ Adjunctive prof., University of Sassari, Italy.

Introduction and aim: Following tooth extraction the alveolar ridge undergoes a remodeling process that can influence implant therapy in the edentulous area. It has been hypothesized that immediate implant placement in fresh extraction sockets may limit the extent of bone remodeling, nevertheless, the literature concerning implants inserted into fresh extraction sockets is controversial. The aim of the present study was to test the hypothesis that there is no difference in clinical, radiographic and aesthetic outcomes positioning single post-extractive $7 \mathrm{~mm}$-diameter implants or waiting 4 months after molar extraction and socket preservation procedure.

Materials and Methods: Patients requiring one implant-supported single restoration to replace a fail- ing tooth in the molar region of both maxilla and mandible were selected. Patients were randomised according to a parallel group design into two arms: implant installation in fresh extraction sockets grafted with cortico-cancellous heterologous bone and porcine derma (group A) or delayed implant installation 4 months after tooth extraction and socket preservation using the same materials (group B). Implants were submerged for 4 months. The primary outcome measures were the success rates of the implants and prostheses and the occurrence of any surgical and prosthetic complications during the entire follow-up. Secondary outcome measures were: peri-implant marginal bone level (MBL) changes, resonance frequency analysis (ISQ) and pink esthetic score (PES) values at implant placement (baseline) up to 1 year after loading.

Group A
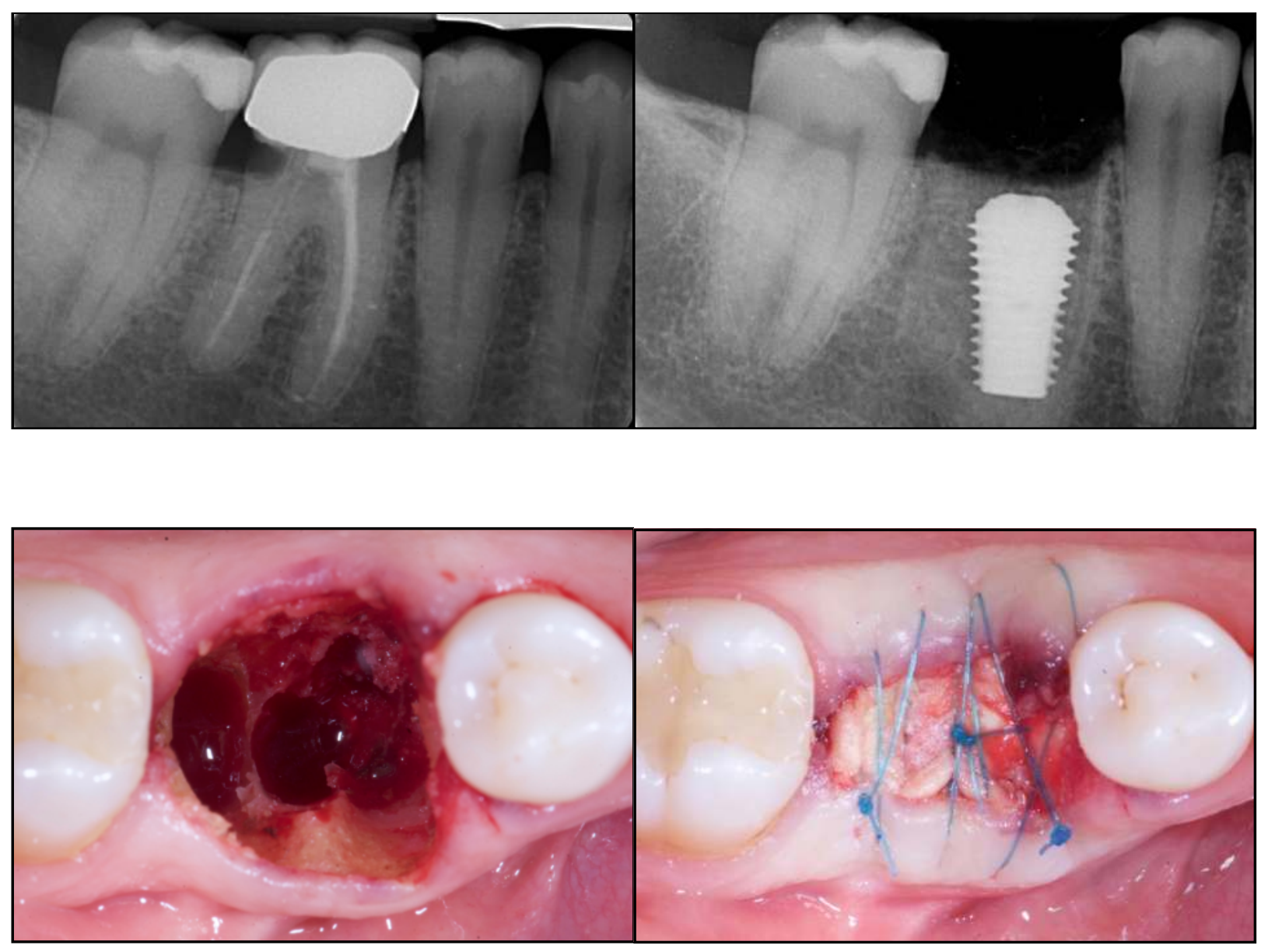

Group B

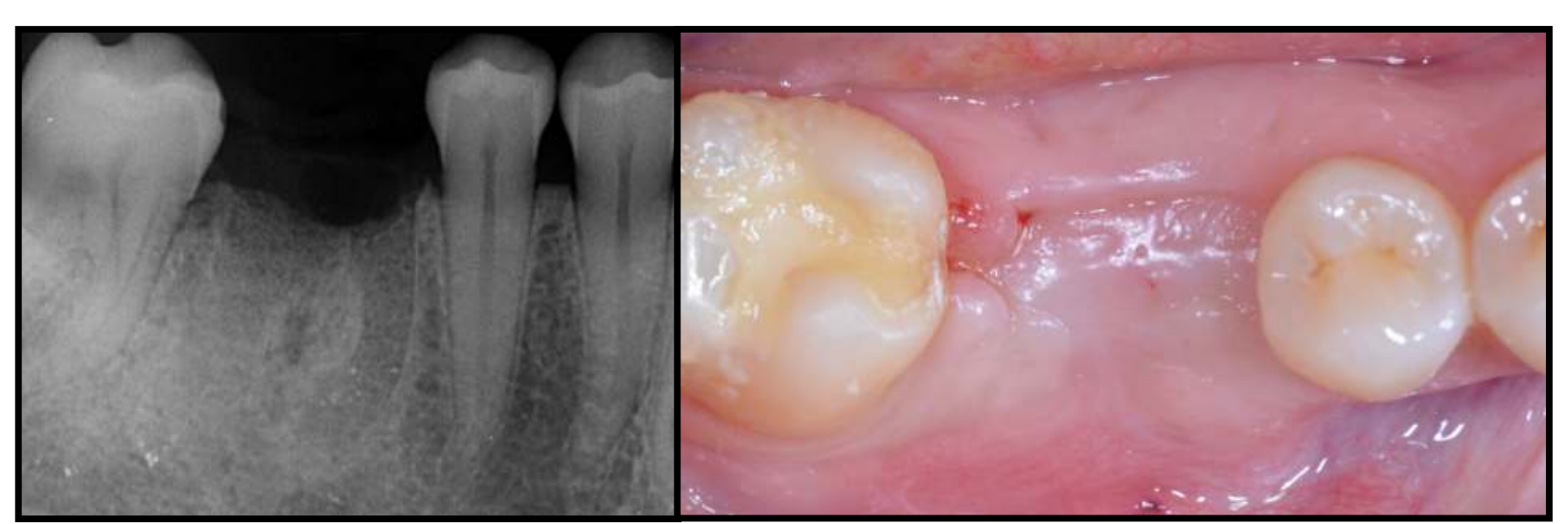

Group B

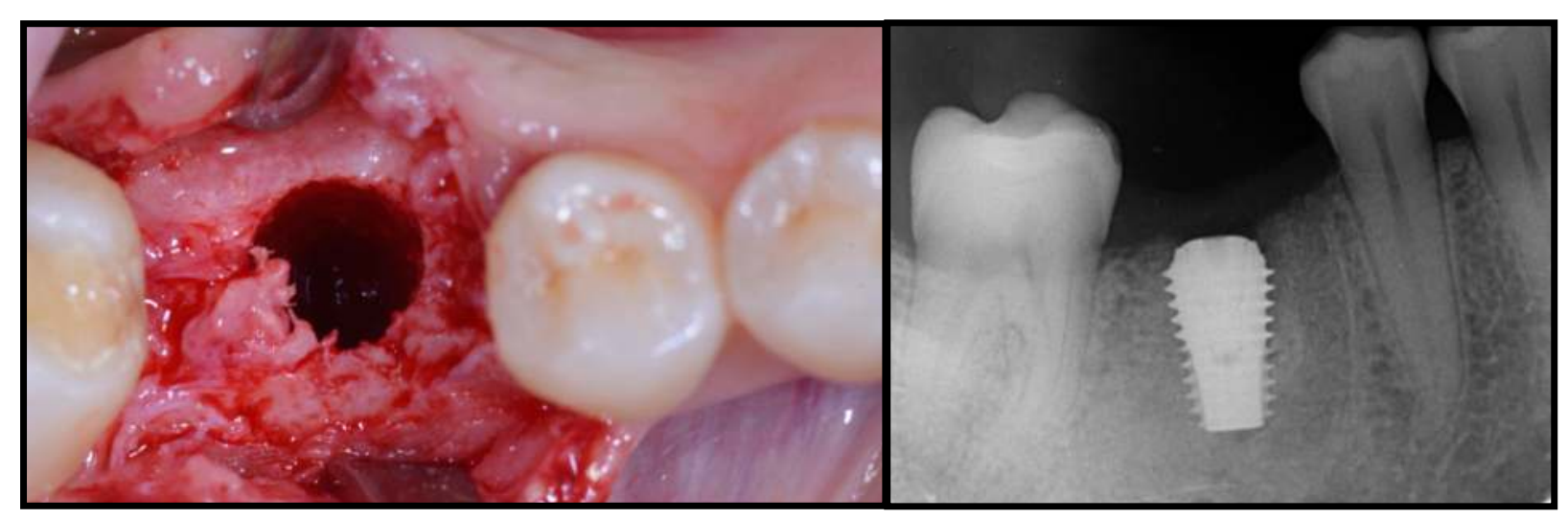

Results: Twelve patients were randomised to group A and 12 to group B. No patient dropped out within 1 year after loading. No implant and prosthesis failed and no complications occurred during the entire follow-up. One year after loading, statistically significant higher mean MBL loss was experienced in group A $(0.63 \mathrm{~mm} \pm 0.31 \mathrm{~mm})$ compared to group B $(0.23 \mathrm{~mm} \pm 0.06 \mathrm{~mm})$; difference $0.41 \mathrm{~mm}(95 \%$ CI $0.17-$ $0.53 ; \mathrm{P}=0.001)$. Six-month after implant placement, mean ISQ value was $78.8 \pm 2.8$ for group A and $79.9 \pm 3.6$ for group B, showing no statistically significant differences between groups (difference 1.1; 95\% CI: 0.04 to $2.96 ; \mathrm{P}=0.422$ ). One year after loading, mean PES was $10.6 \pm 1.8$ [range: 8 to 13 ] in group A and $12.2 \pm 1.2$ [range: 11 to 14 ] in group B. The difference was statistically significant $(1.6 \pm 2.7 ; 95 \% \mathrm{CI}$ $-0.55-2.55 ; \mathrm{P}=0.019)$ with better results for group $\mathrm{B}$.

Group A

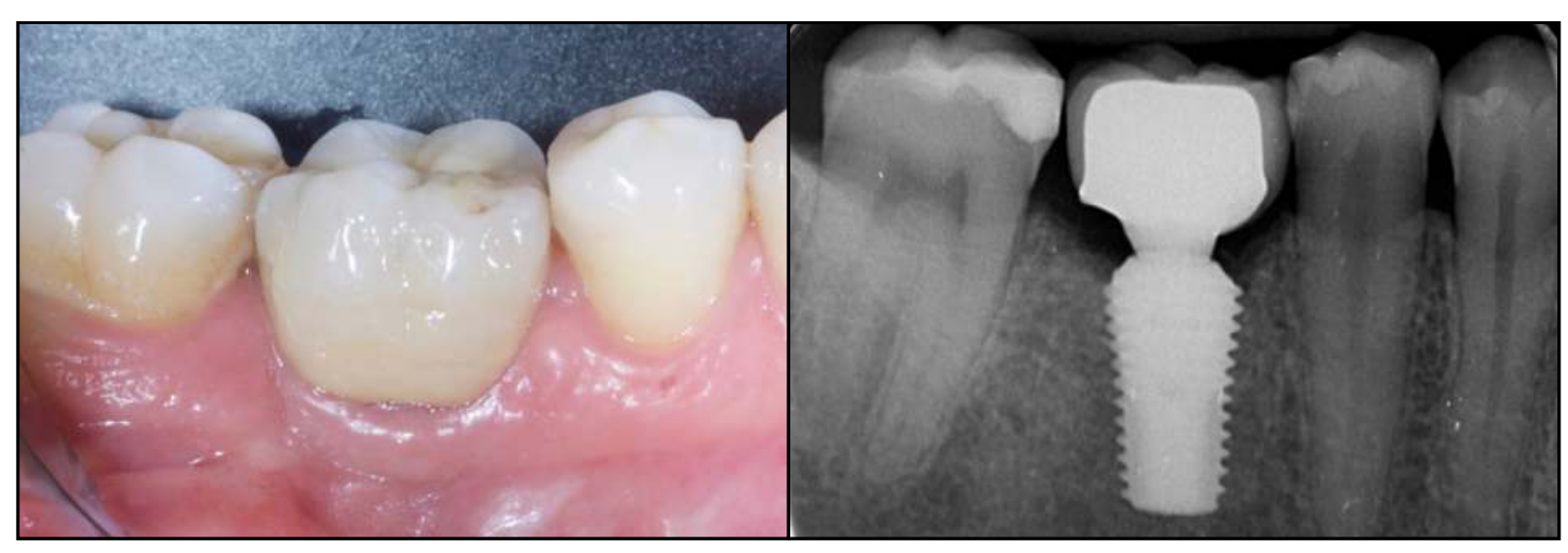

Group B

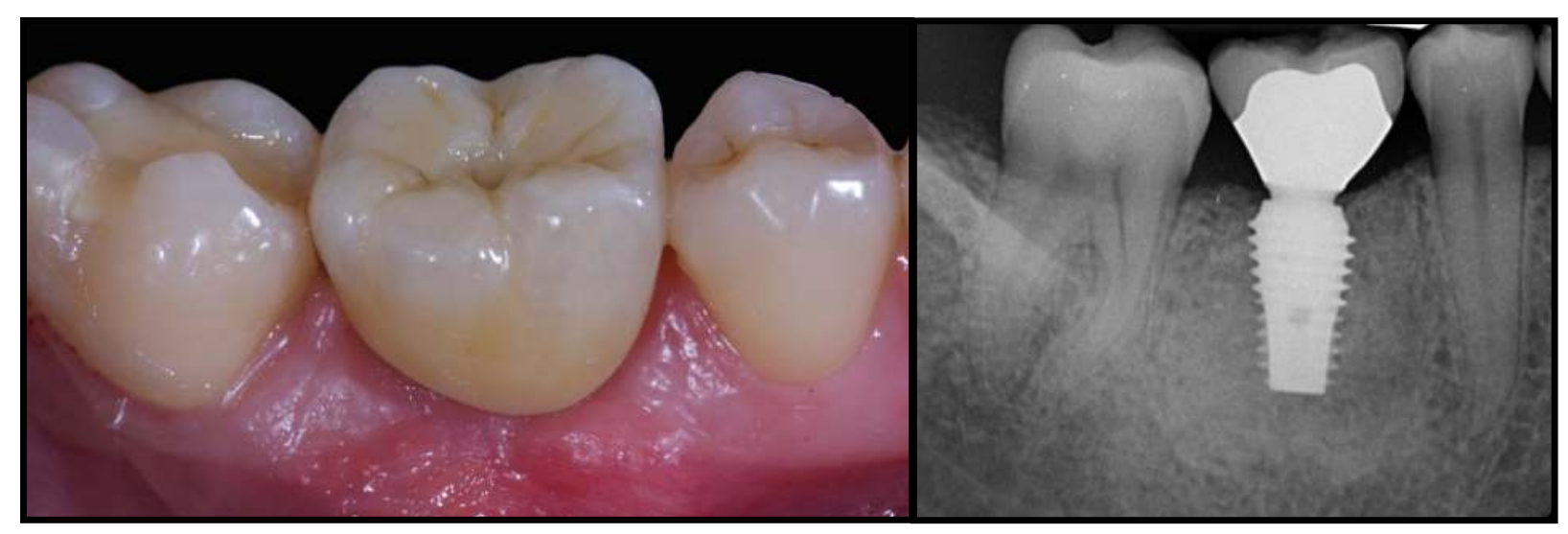

Conclusions: Within the limitations of this study, both procedures achieved successful results over the 1-year follow-up period, but waiting 4 months after tooth extraction and socket preservation procedure was associated with less marginal bone loss and a better aesthetic outcome.

Schropp L, Wenzel A, Kostopoulos L, Karring T. Bone heal- ing and soft tissue contour changes following single-tooth extraction: a clinical and radiographic 12-month prospective study. Int J Periodontics Restorative Dent 2003;23:313-323.

Meloni SM, Tallarico M, Lolli FM, Deledda A, Pisano M, Jovanovic SA. Postextraction socket preservation using epithelial connective tissue graft vs porcine collagen matrix. 1-year results of a randomised controlled trial. Eur J Oral Implantol 2015;8:39-48.

Esposito M, Grusovin MG, Polyzos IP, Felice P, Worthington HV. Timing of implant placement after tooth extraction: immediate, immediate-delayed or delayed implants? A Cochrane systematic review. Eur J Oral Implantol 2010;3: 189-205.

Tallarico M, Xhanari E, Pisano M, De Riu G, Tullio A, Meloni SM. Single post-extractive ultra-wide 7mm-diameter implants versus implants placed in molar healed sites after socket preservation for molar replacement: 6-month post- loading results from a randomised controlled trial. Eur J Oral Implantol. 2016;9:263-275. 